\title{
Recoverable Encryption through Noised Secret over Large Cloud
}

\section{Sushil Jajodia ${ }^{1}$, W. Litwin² \& Th. Schwarz ${ }^{3}$}

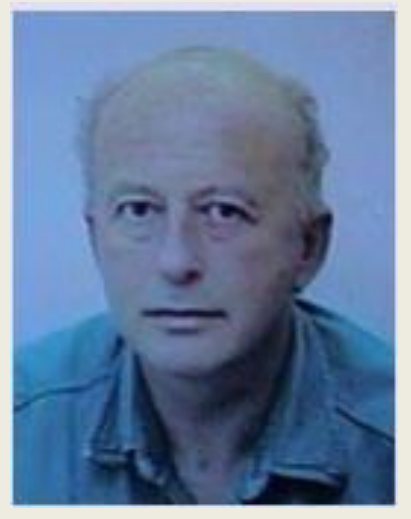

${ }^{1}$ George Mason University, Fairfax, VA \{jajodia@gmu.edu\}

2Université Paris Dauphine, Lamsade \{witold.litwin@dauphine.fr\}

${ }^{3}$ Thomas Schwarz, UCU, Montevideo \{tschwarz@ucu.edu.uv\} 


\section{What?}

- New schemes for backup of encryption keys entrusted to an Escrow

- Collectively called $\mathrm{RE}_{\mathrm{NS}}$ Schemes

-They backup high quality encryption keys

-AES (256b), DH 500+b...

- Backup itself is specifically encrypted

- Unlike a traditional simple key copy 


\section{What?}

- Fast brute-force recovery remains possible

- In the absence of key owner

- Within the timing wished by the recovery requestor

- But only over a large cloud

$1 \mathrm{~K}-100 \mathrm{~K}$ nodes 


\section{What?}

- Unwelcome recovery is unlikely

- E.g. could easily take, say, 70 or even 700 days at escrow's processor alone

- Illegal use of a large cloud is implausible

- Cloud providers do best to prevent it - Easily noticeable if ever starts

-Follow the money

- Leaves compromising traces in numerous logs 
- High quality key loss danger is Achilles' heel of modern crypto

-Makes many folks refraining of any encryption

-Other loose many tears if unthinkable happens 


\section{Why}

- If you create key copies...

- Every copy increases danger of disclosure

-For an Escrow, her/his copy is an obvious temptation

- Some Escrows may not resist to

- In short users face the dilemma:

Key loss or disclosure? That is The

Question 
- $R E_{N S}$ schemes alleviate this dilemma

- Easily available large clouds make them realistic

- Our schemes should benefit numerous applications 


\section{How (Overview) : Key Owner Side}

- Key owner or client chooses

inhibitive timing of 1-node (brute-

force) recovery

- Presumably unwelcome at escrow's site alone

-E.g. 70 days

- Or 700 days for less trusted escrows

- Or anything between 


\section{How : Key Owner Side}

- Consequently, the owner fixes a large integer

-Called backup encryption complexity or hardness

- Actually, this step may be programmed

- The backup encryption agent on client node may be in charge of 


\section{How : Key Owner Side}

- Key owner or the agent creates

the shared noised secret

- Some share(s) of the actual secret become noised shares

- "Burried » among very many look-alike but fake noise shares 


\section{How : Key Owner Side}

- The only way to recognize

whether a noise share is a noised

one is to try out its "footprint"

- The owner/agent creates the footprint for each noised share

- Each footprint is unique

- Remember Cinderella? 


\section{How : Key Owner Side}

- Key owner/agent sends the noised secret to Escrow

- Noised secret is the backup

- Guess your key by its print in this mess (inspired by CSIS actual ex.)

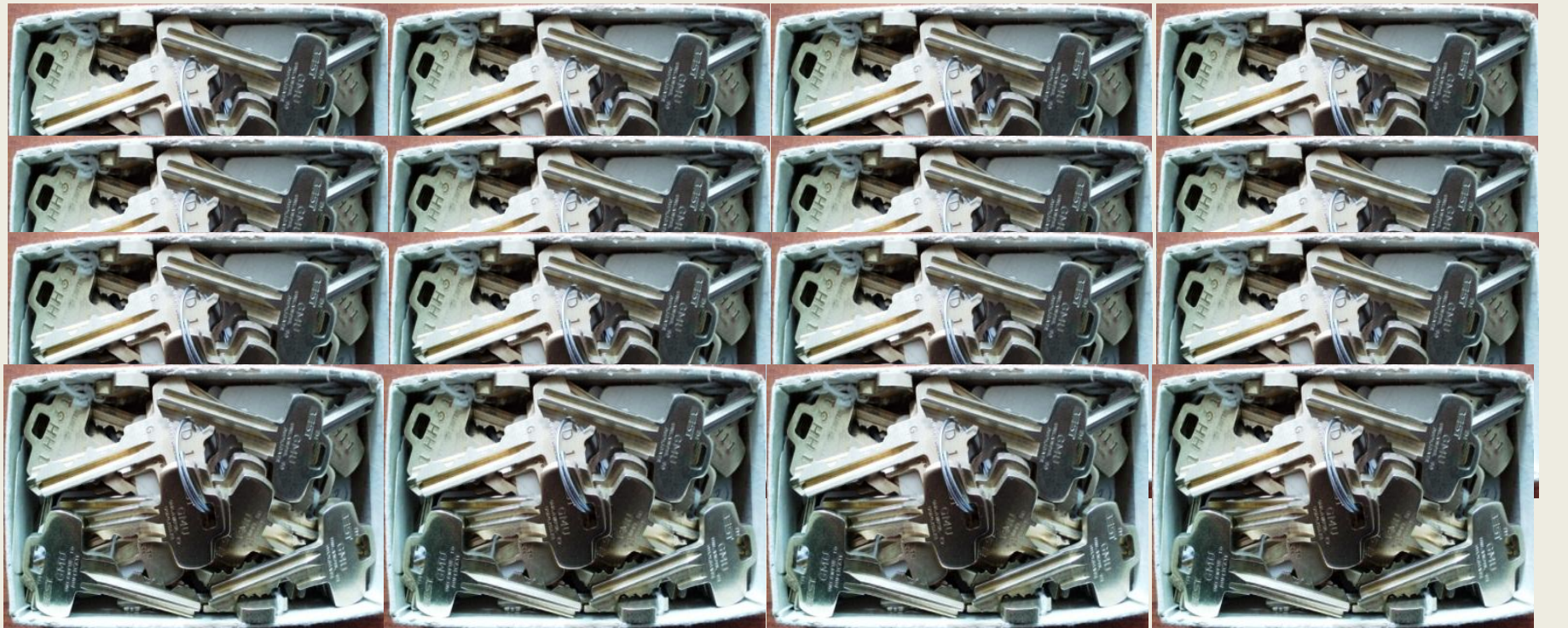




\section{How (Overview) : Escrow Side}

- Key requestor asks Escrow to recover data in acceptable max recovery time -E.g. 10 min

- Escrow's server sends the time and all but one shares of the noised secret to the cloud

- Intruder to the cloud cannot find the key 


\section{How : Escrow's Side}

- $\mathrm{RE}_{\mathrm{NS}}$ scheme executed at the cloud chooses the cloud size

-To fit the calculus time limit for sure

- Say 10K nodes

- Search for the noised share gets partitioned over the nodes

- Nodes work in parallel

- Matching the "footprints" 


\section{How : Escrow's Side}

- Every lucky node reports back to

Escrow the noised share found

- Escrow' server recovers the key from all the shares

- Using the clasical XORing

- Sends the recovered key to

Requestor

-Not forgetting the bill 


\section{What Else?}

- Well, everything is in details

-Client Side Encryption

-Server Side Recovery

- Static Scheme

- Scalable Scheme

-Related Work

-Conclusion 


\section{What Else?}

- More :

- Res. Rep.

http://www.lamsade.dauphine.fr/ litwin/Recoverabl e\%20Encryption 10.pdf

-S. Jajodia, W. Litwin \& Th. Schwarz. Recoverable Encryption through a Noised Secret over a Large Cloud.

- 5th Inl. Conf. on Data Management in Cloud, Grid and P2P Systems (Globe 2012 )

- Publ. Springer Verlag, Lecture Notes in Comp. 


\section{Client Side (Backup) Encryption}

- Client $X$ backs up encryption key $S$

- $X$ estimates 1-node inhibitive time

$D$

-Say 70 days

- $D$ measures trust to Escrow

-Lesser trust?

- Choose 700 days 


\section{Client Side Encryption}

- $D$ determines minimal cloud size $N$ for future recovery in any acceptable time $R$

-Chosen by recovery requestor

- E.g. $10 \mathrm{~min}$

$-X$ expects $N>D / R$ but also $N \cong D / R$

- E.g. $N \cong 10 \mathrm{~K}$ for $D=70$ days

$-N \cong 100 K$ for $D=700$ days 


\section{Client Side Encryption}

- $X$ creates a classical shared secret for $S$

$-S$ is seen as a large integer,

-E.g., 256b long for AES

-Basically, $X$ creates a 2-share secret

-Share $s_{0}$ is a random integer

- Share $s_{1}$ is calculated as $s_{1}=s_{0}$ XOR $S$

Common knowledge:

$-S=s_{0}$ XOR $s_{1}$ 


\section{Client Side Encryption}

- $X$ transforms the shared secret into a noised one

- $X$ makes $s_{0}$ a noised share :

- Chooses a 1-way hash $H$

- E.g. SHA 256

- Computes the hint $h=H\left(s_{0}\right)$

- Chooses the noise space

$I=0,1 . . . m, . . M-1$

- For some large $M$ determined as we explain soon 


\section{Client-Side Encryption}

- Each noise $m$ and $s_{0}$ define a noise share $s$

- In a way we show soon as well

- There are $M$ different pseudo random noise shares

- All but one are different from $s_{0}$

- But it is not known which one is $s_{0}$

- The only way to find for any $s$ whether $s=s_{0}$ is to attempt the match

$$
H(s) ?=h
$$




\section{Shared Secret / Noised (Shared) Secret}

$$
S=S_{1} \text { XOR } S_{0}
$$

Hint $H\left(s_{0}\right)$

$S=S_{1}$

$H$ is one-way hash SHA 256 by default 


\section{Client Side Encryption}

- $X$ estimates the 1-node throughput $T$

- \# of match attempts $H(s)$ ? $=h$ per time unit

- 1 Sec by default

- $X$ sets $M$ to $M=\operatorname{lnt}(D T)$.

$-M$ should be $2^{40} \div 2^{50}$ in practice 


\section{Client Side Encryption}

- $X$ randomly chooses $m \in I=[0,1 \ldots M[$

- Calculates base noise share $f=s_{0}-m$

- Defines noised share $s_{0}{ }^{n}=(f, M, h)$.

- Sends the noised secret $S^{\prime}=\left(s_{0}{ }^{n}, s_{1}\right)$ to

Escrow as the backup 
Escrow-Side Recovery (Backup Decryption)

- Escrow $E$ receives legitimate request of $S$ recovery in time $R$ at most

- E chooses between static or scalable recovery schemes

- $E$ sends data $S^{\prime \prime}=\left(s_{0}{ }^{n}, R\right)$ to some cloud node with request for processing accordingly

-Keeps $s_{1}$ out of the cloud 


\section{Recovery Processing Parameters}

- Node load $L_{n}$ : \# of noises among $M$ assigned to node $n$ for match attempts

- Throughput $T_{n}$ : \# of match attempts node $n$ can process / sec

- Bucket (node) capacity $B_{n}$ : \# of match attempts node $n$ can process / time $R$ $-B_{n}=R T_{n}$

- Load factor $\alpha_{n}=L_{n} / B_{n}$ 


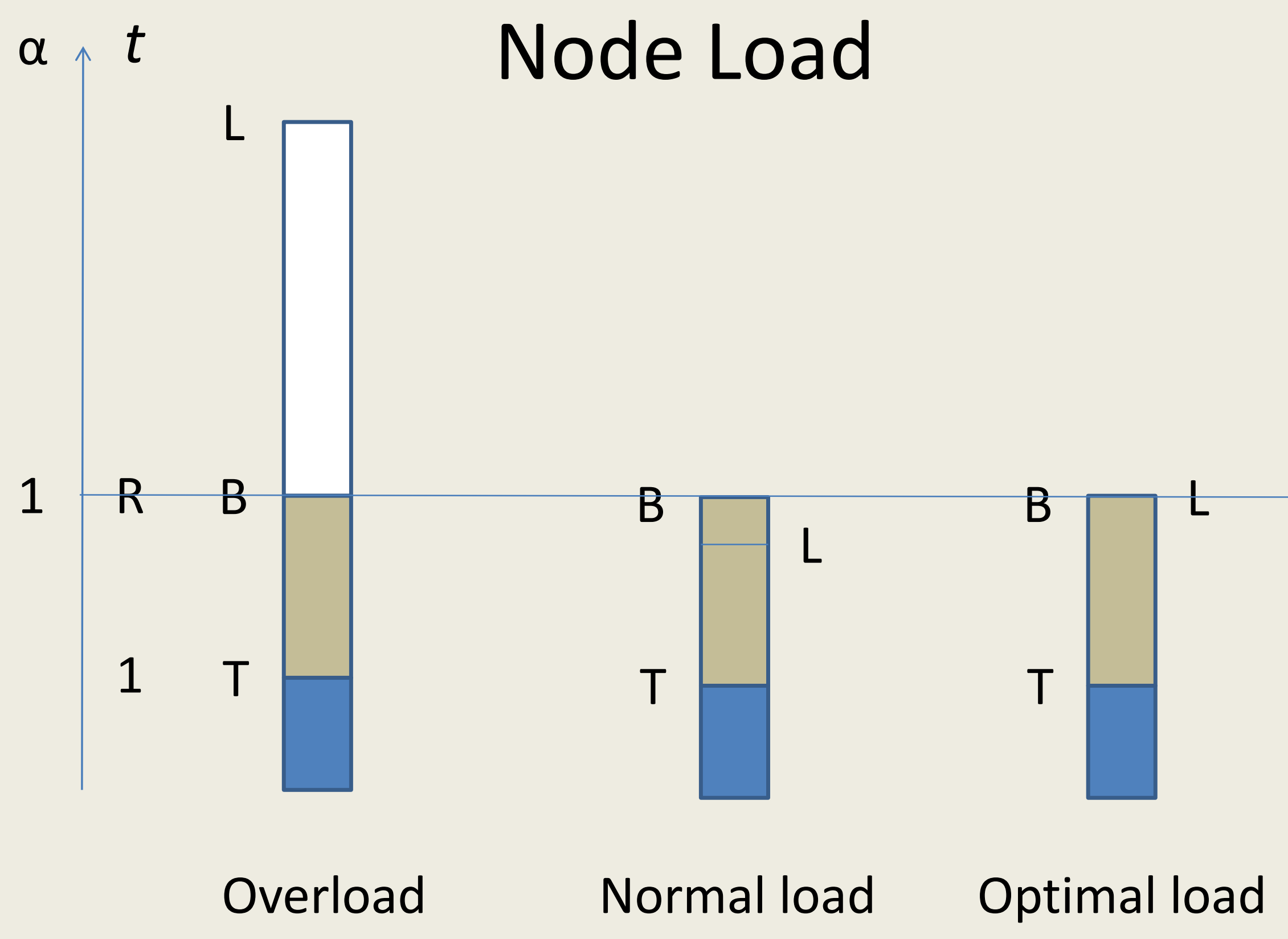




\section{Recovery Processing Parameters}

- Notice the data storage oriented vocabulary

- Node $n$ respects $R$ iff $\alpha_{n} \leq 1$

-Assuming $T$ constant during the processing

- The cloud respects $R$ if for every $n$ we have $\alpha_{n} \leq 1$

- This is our goal

-For both static and scalable schemes we now present 


\section{Static Scheme}

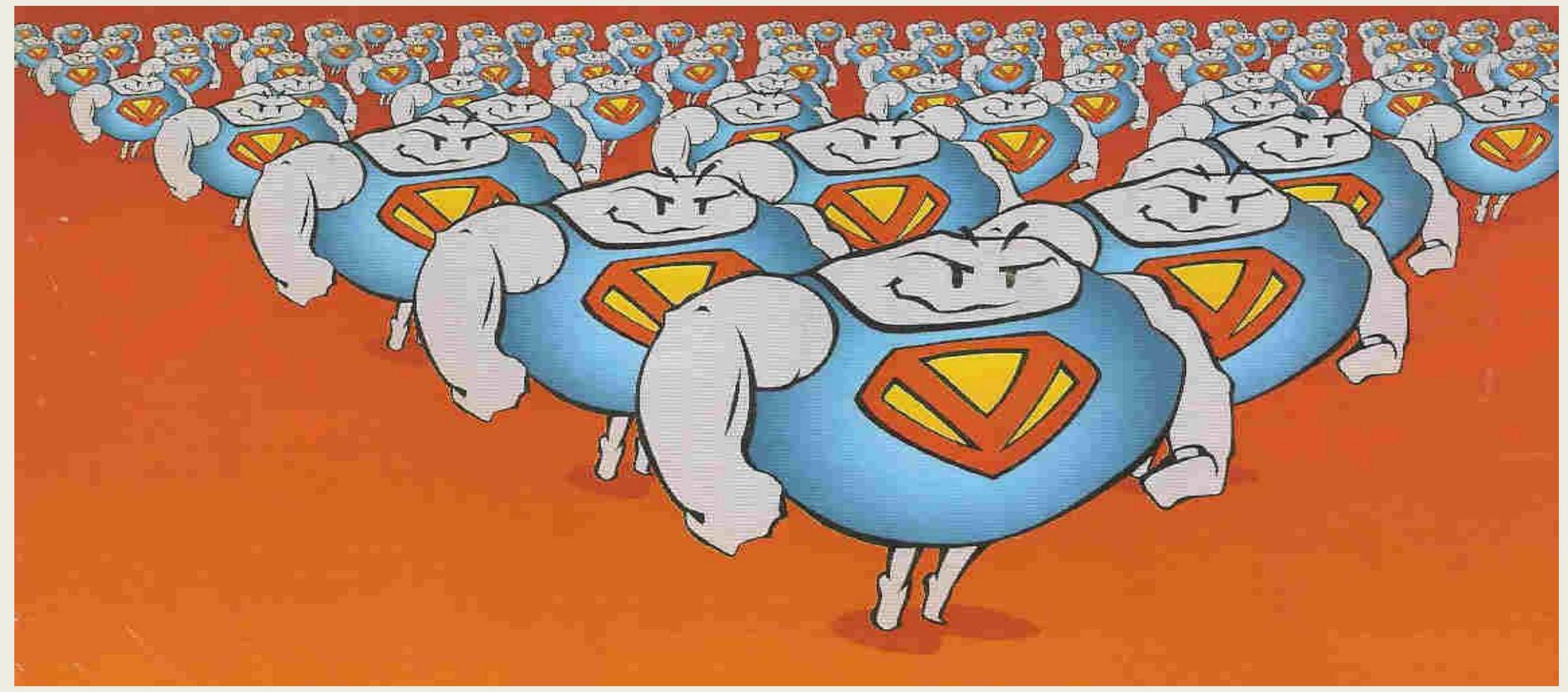

- Intended for a homogenous Cloud

- All nodes provide the same throughput 


\section{Static Scheme : Init Phase}

- Node $C$ that got $S^{\prime \prime}$ from $E$ becomes coordinator

- Calculates $a(M)=M / B(C)$

-Usually $\alpha(M) \gg>1$

- Defines $N$ as $\lceil a(M)\rceil$

-Implicitly considers the cloud as homogenous

- E.g., $N=10 \mathrm{~K}$ or $N=100 \mathrm{~K}$ in our ex. 


\section{Static Scheme : Map Phase}

- $C$ asks for allocation of $N-1$ nodes

- Associates logical address $n=1,2 \ldots N-1$ with each new node \& 0 with itself

- Sends out to every node $n$ data $\left(n, a_{0}, P\right)$

$-a_{0}$ is its own physical address, e.g., IP

$-P$ specifies Reduce phase 


\section{Static Scheme : Reduce Phase}

- $P$ requests node $n$ to attempt matches for every noise share $s=(f+m)$ such that $n=m \bmod N$

- In practice, e.g., while $m<M$ :

- Node 0 loops over noise $m=0, N, 2 N \ldots$

- So over the noise shares $f, f+N, f+2 N$...

- Node 1 loops over noise $m=1, N+1,2 N+1 \ldots$

- Node $N-1$ loops over $m=$ (your guess here) 


\section{Static Scheme : Node Load}

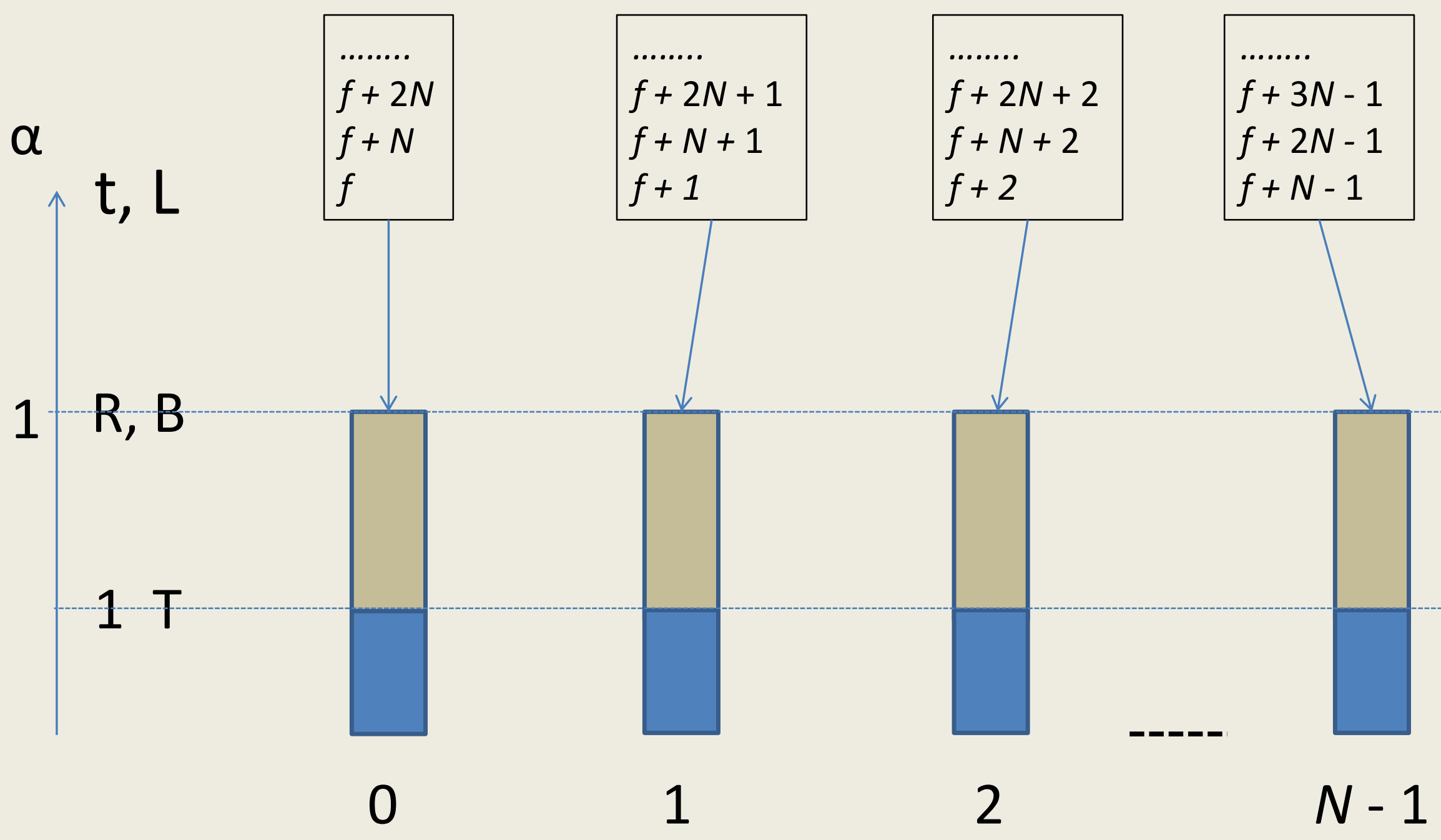




\section{Static Scheme}

- Node $n$ that gets the successful match sends $s$ to $C$

- Otherwise node $n$ enters Termination

- C asks every node to terminate

- Details depend on actual cloud

- $C$ forwards $s$ as $s_{0}$ to $E$ 


\section{Static Scheme}

- $E$ discloses the secret $S$ and sends $S$ to Requestor

- Bill included (we guess)

- E.g., up to $400 \$$ on CloudLayer for

$-D=70$ days

$-R=10 \mathrm{~min}$

- Both implied $N=10 \mathrm{~K}$ with private option 


\section{Static Scheme}

- Observe that $N \geq D / R$ and $N \cong D / R$

- If the initial estimate of $T$ by $S$ owner holds

- Observe also that for every node $n$, we have $\alpha(n) \leq 1$

- Under our assumptions maximal recovery

time is thus indeed $R$

- Average recovery time is $R / 2$

-Since every noise share is equally likely to be the lucky one 


\section{Static Scheme}

- See papers for

-Details,

-Numerical examples

- Proof of correctness

- The scheme really partitions I

-Whatever is $N$ and $s_{0}$, one and only one node finds $s_{0}$ 


\section{Static Scheme}

- Safety

-No disclosure method can in practice be faster than the scheme

-Dictionary attack, inverted file of hints...

- Other properties 


\section{Scalable Scheme}

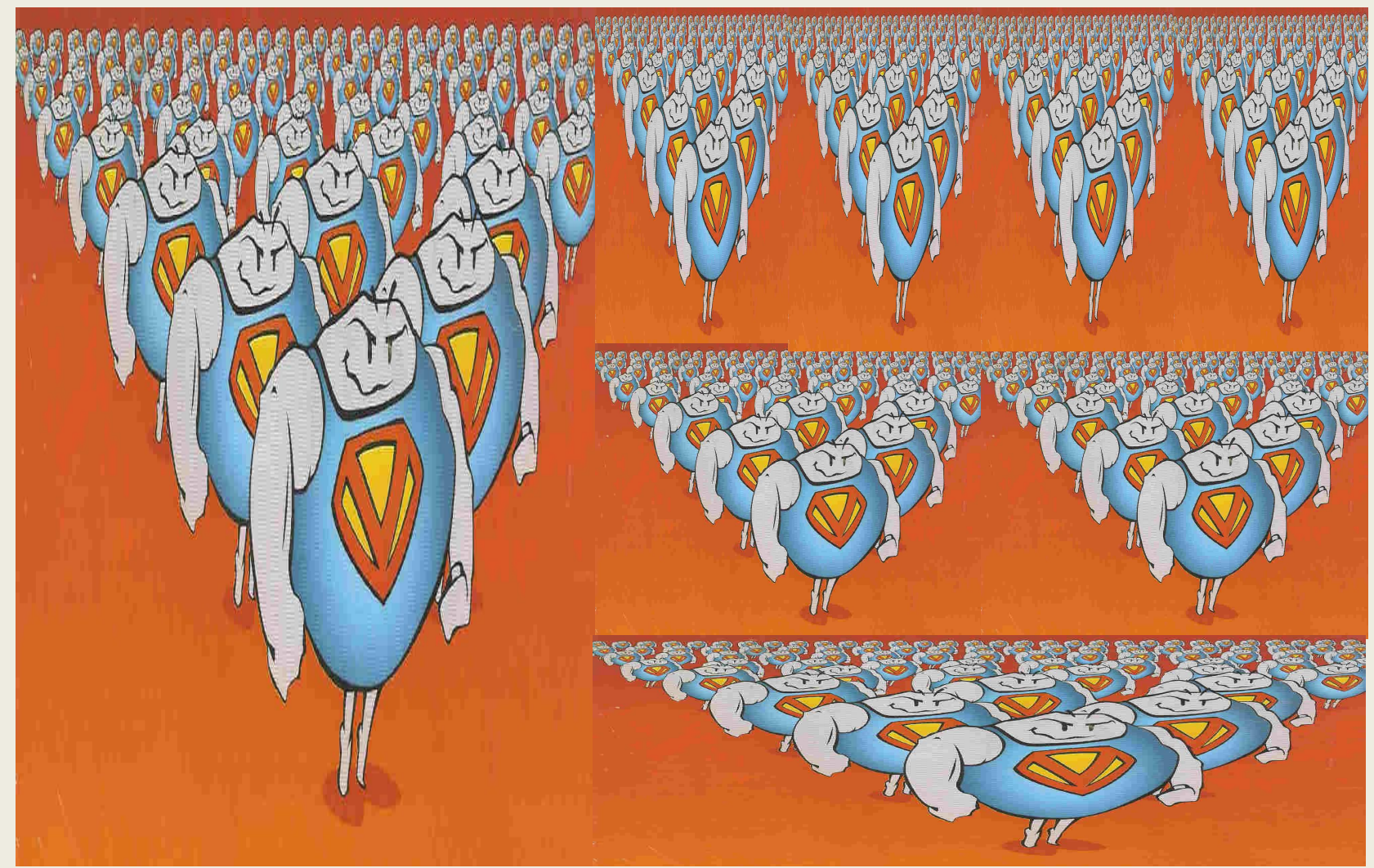

- Heterogeneous cloud

- Node throughputs may differ 


\section{Scalable Scheme}

- Intended for heterogenous clouds

- Different node throughputs

- Basically only locally known

- E.g.

-Private or hybrid cloud

-Public cloud without so-called private node option 


\section{Scalable Scheme}

- Init phase similar up to $\alpha(M)$ calculus

- Basically $\alpha(M)>>1$

- Also we note it now $\alpha_{0}$

- If $\alpha>1$ we say that node overflows

- Node 0 sets then its level $j$ to $j=0$ and splits

- Requests node $2^{j}=1$

- Sets $j$ to $j=1$

- Sends to node $1,\left(S^{\prime \prime}, j, a_{0}\right)$ 


\section{Scalable Scheme}

- As result

- There are $N=2$ nodes

- Both have $j=1$

- Node 0 and node 1 should each process $M / 2$ match attempts

- We show precisely how on next slides

- Iff both $\alpha_{0}$ and $\alpha_{1}$ are no more than 1

- Usually it should not be the case

- The splitting should continue as follows 


\section{Scalable Scheme}

- Recursive rule

- Each node $n$ splits until $\alpha_{n} \leq 1$

- Each split increases node level $j_{n}$ to $j_{n}+1$

- Each split creates new node $n^{\prime}=n+2^{j_{n}}$

- Each node $n^{\prime}$ gets $j_{n^{\prime}}=j_{n}$ initially

- Node 0 splits thus perhaps into nodes $1,2,4 \ldots$

- Until $\alpha_{0} \leq 1$

- Node 1 starts with $j=1$ and splits into nodes

$3,5,9 \ldots$

- Until $\alpha_{1} \leq 1$ 


\section{Scalable Scheme}

- Node 2 starts with $j=2$ and splits into

$6,10,18 \ldots$

- Until $\alpha_{2} \leq 1$

- Your general rule here

- Node with smaller $T$ splits more times and vice versa 


\section{Scalable Scheme : Splitting}

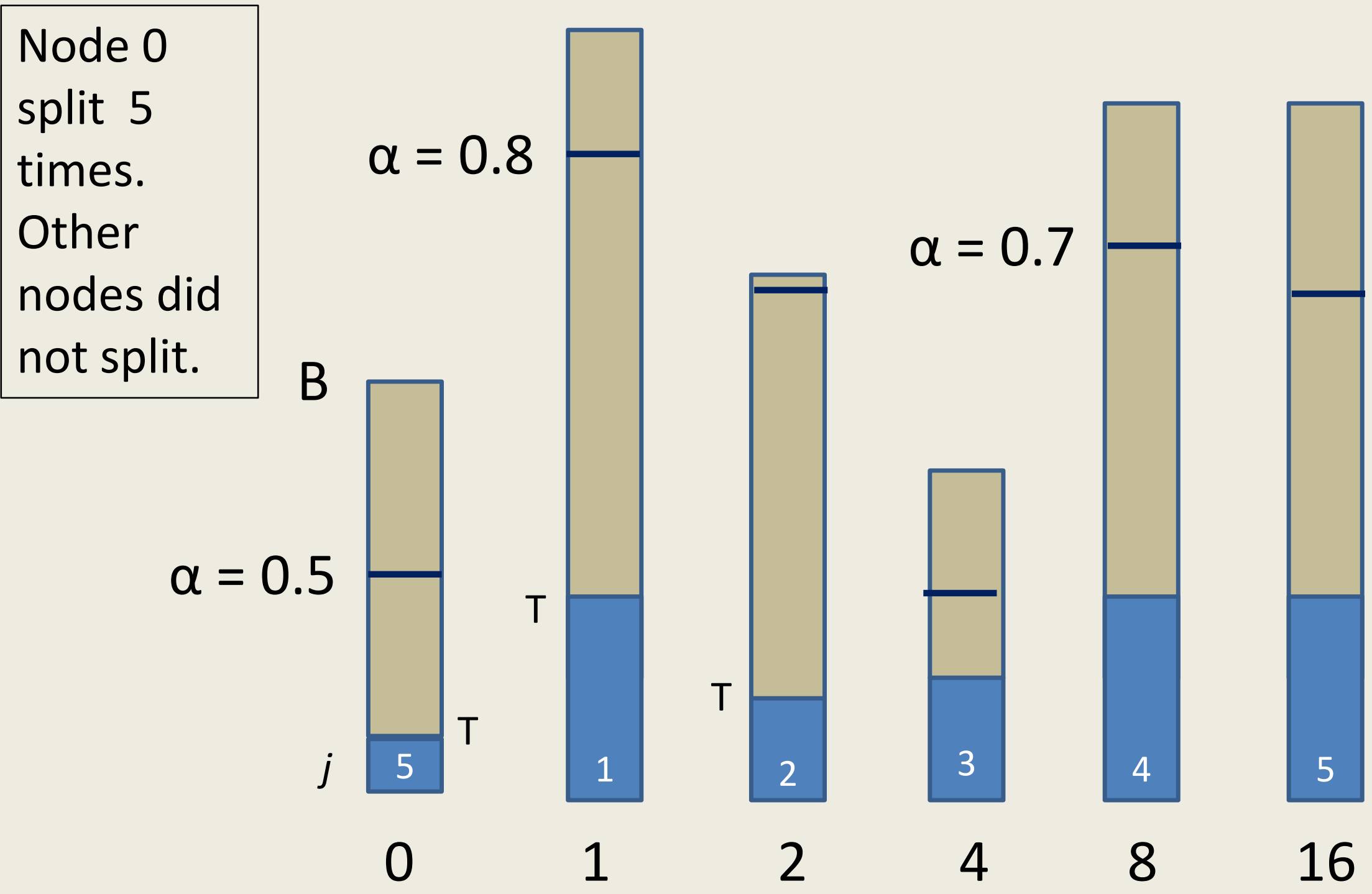




\section{Scalable Scheme}

- If cloud is homogenous, the address space is contiguous

- Otherwise, it is not

- No problem

- Unlike for a extensible or linear hash data structure 


\section{Scalable Scheme : Reduce phase}

- Every node $n$ attempts matches for every noise $k \in[0, \mathrm{M}-1]$ such that $n=k \bmod 2^{j_{n}}$.

- If node 0 splits three times, in Reduce phase it attempts to match noised shares $(f+k)$ with $k=0,8,16 \ldots$

- If node 1 splits four times, it attempts to match noised shares $(f+k)$ with $k=1,17$, 33...

- Etc. 


\section{Scalable Scheme : Reduce Phase}

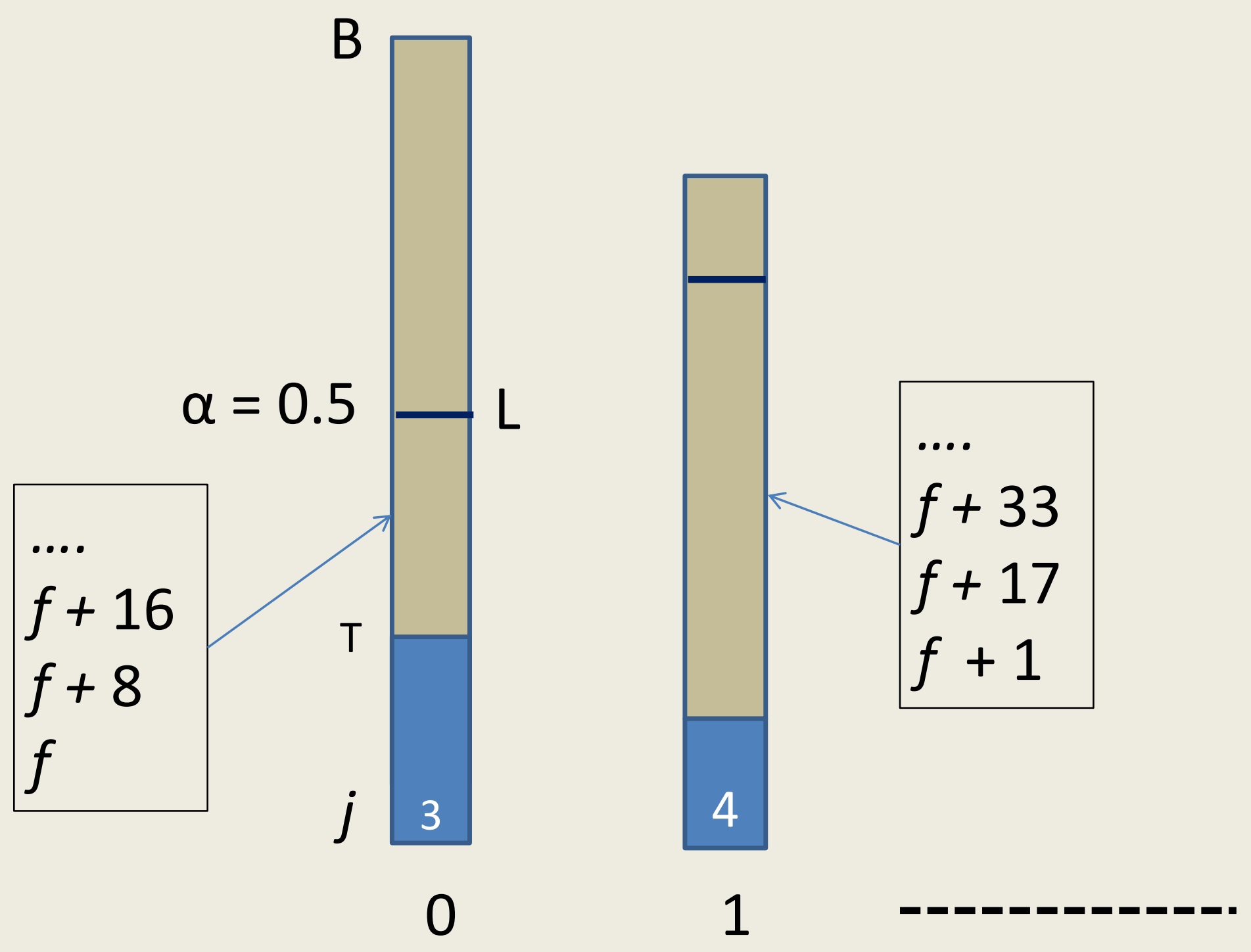




\section{Scalable Scheme}

- $N \geq D / R$

- If $S$ owner initial estimate holds

- For homogeneous cloud it is $30 \%$ greater on the average and twice as big at worst / static scheme

- Cloud cost may still be cheaper - No need for private option

- Versatility may still make it preferable besides 


\section{Scalable Scheme}

- Max recovery time is up to $R$

- Depends on homogeneity of the cloud

- Average recovery time is up to $R / 2$

- See again the papers for

- Examples

- Correctness

- Safety

-Detailed perf. analysis remains future work 


\section{Related Work}

- RE scheme for outsourced $\mathrm{LH}^{*}$ files

- CSCP scheme for outsourced LH* records sharing

- Crypto puzzles

- One way hash with trapdoor

- 30-year old excitement around Clipper chip

- Botnets 


\section{Conclusion}

- Key safety is Achilles' heel of cryptography

- Key loss or key disclosure ? That is The Question

- $\mathrm{RE}_{\mathrm{NS}}$ schemes alleviate the dilemma

- Future work Deeper formal analysis

-Proof of concept implementation

-Variants 


\section{Thanks}

for

Your Attention

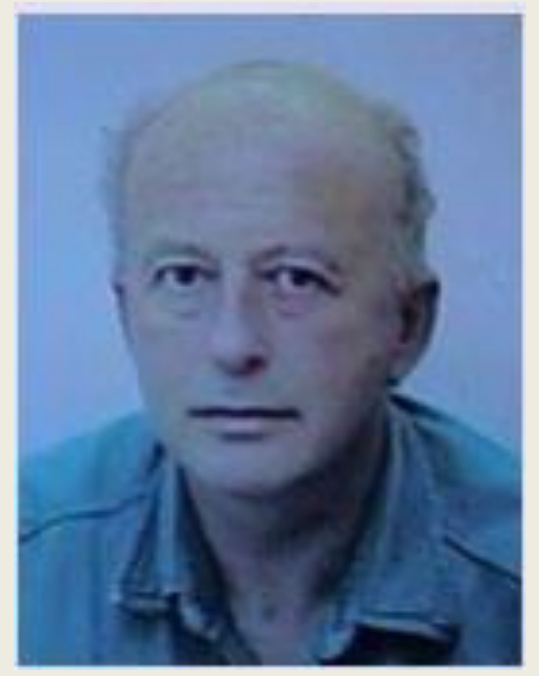

Witold LITWIN \& al 